\title{
Achieving junction stability in heavily doped epitaxial Si:P
}

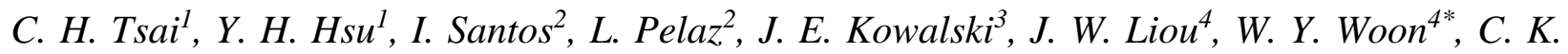
$\operatorname{Le}^{1^{*}}$

${ }^{1}$ Institute of Applied Mechanics, National Taiwan University, No. 1, Sec. 4, Roosevelt Rd., Taipei, 10617, Taiwan

${ }^{2}$ Department of Electronics, University of Valladolid, Paseo de Belen 15, 47011 Valladolid, Spain.

${ }^{3}$ DSG Technologies, Inc. 2372 Qume Drive Suite F San Jose, CA 95131 USA

${ }^{4}$ Department of Physics, National Central University, No. 300, Jungda Road, Jungli, 32054, Taiwan

Junction stability and donor deactivation in silicon at high doping limit has been a longstanding issue in advanced semiconductor devices. Recently, heavily doped epitaxial Si:P layer with phosphorus concentrations as high as $3 \times 10^{21}$ at. $/ \mathrm{cm}^{3}$ has been employed in nanowire field-effect transistor (FET) devices for sub-3 nm technology node as low resistance sourcedrain and channel stressor. In such highly doped Si:P, the actual dopant activation is much less than nominal phosphorus concentration due to inactive phosphorus atoms arising from dopantvacancy defects $\left(\mathrm{P}_{\mathrm{n}} \mathrm{V}\right)$ clustering phenomenon. Even with state-of-the-art high temperature millisecond annealing, this epitaxial film is thermally unstable upon subsequent thermal treatments. To overcome this limitation, we demonstrate a selective dopant activation scheme 
which results from the dipole moments of inactive $\mathrm{P}_{\mathrm{n}} \mathrm{V}$ structures within the crystal lattice and their direct energy coupling with the external electric field. It's found that superior stability in dopant activation can be achieved through microwave annealing when a specific temperature and field conditions are met using a triple-parallel-susceptor setup in the microwave cavity. Based on experimental results and ab-initio calculation, we proposed a model, whereas the microwave- $\mathrm{P}_{\mathrm{n}} \mathrm{V}$ interactions result in a specific distribution of dopant defect dominated by thermally stable $\mathrm{P}_{4} \mathrm{~V}$ clusters through elimination of unstable low order $\mathrm{P}_{\mathrm{n}} \mathrm{V}$, leading to the suppression of donor deactivation and achieving thermally stable junction. 
Silicon is considered as one of the most technologically relevant materials today and its continuing development is the cornerstone of technological innovation such as artificial intelligence and next generation wireless communication. The speed of electronic devices relies on the free carrier concentration available in the semiconductor. The generation of free carriers requires achieving high concentration of electrically active dopants, typically controlled by doping and annealing techniques. The selective growth of in-situ doped epitaxial $\mathrm{Si}: \mathrm{P}^{1-4}$ is a major innovation that can significantly reduce source-drain external resistance and simultaneously act as stressor for advanced field effect transistor options beyond 3nm including gate-all-around (GAA) nanowire and FinFET devices. ${ }^{5-7}$ The continuing device scaling demands for ever-increasing phosphorus concentration $[\mathrm{P}]$ in epitaxial Si:P. There has been significant efforts to increase phosphorus concentration $[\mathrm{P}]$ in epitaxial Si:P, and the highest $[\mathrm{P}]$ achieved nowadays is about $3 \times 10^{21}$ at. $/ \mathrm{cm}^{3}$ by state-of-the-art epitaxial Si:P processes, virtually limited by its solid solubility. However, actual dopant activation is often much less than nominal phosphorus concentration due to a large percentage of inactive phosphorus atoms arising from dopant - vacancy cluster formation. The $\mathrm{P}$ atoms in phosphorus-vacancy $\left(\mathrm{P}_{\mathrm{n}} \mathrm{V}\right)$ complexes are inactive because the $\mathrm{P}$ atom bounded to vacancy forms an E-center that acts an acceptor level, and counters the doping effect. ${ }^{4}$ These impurity clusters have to be electrically activated through annealing, typically at temperature $>1100 \operatorname{deg} \mathrm{C}$, in particular for nano-structures due to its higher dopant activation energy, ${ }^{8}$ to provide free carrier in the semiconductor.

However, dopant deactivation in highly doped $\mathrm{Si}$ has been a long-standing issue in semiconductor devices application. ${ }^{9-17}$ Such junction instability issue is particularly severe for highly doped Si:P source/drain stressors in nanowire devices with $\mathrm{P}$ concentration $>2 \times 10^{21}$ at. $/ \mathrm{cm}^{3}$. Dhayalan and Chen et $a l^{4,17}$ noted that in a highly doped Si:P layer with $\mathrm{P}$ concentration $>1 \times 10^{21}$ 
at. $/ \mathrm{cm}^{3}$, the dopant activation level was only about $2 \times 10^{20}$ at. $/ \mathrm{cm}^{3}$ due to the large amount of vacancy centered dopant clusters $\left(\mathrm{P}_{\mathrm{n}} \mathrm{V}, \mathrm{n}=1-4\right)$ that are electrically inactive. Subjecting the asdeposited epitaxial layer to a high temperature millisecond annealing (MSA) process such as from laser or flash lamp, ${ }^{18}$ it was found that a fraction of the $\mathrm{P}$ atoms can be activated and can result in an increase in electrical conductivity. ${ }^{4}$ However, it was observed that these activated dopants were thermally unstable and tended to deactivate significantly upon subsequent thermal processing at temperature range between 600 to $700 \mathrm{deg} \mathrm{C}$. The deactivation mechanism of the group $\mathrm{V}$ donors in heavily doped silicon have been reported in previous studies. ${ }^{15-17}$ However, an annealing solution to avoid the dopant deactivation phenomenon has been lacking.

In this work we address the above issue by proposing a selective activation scheme through triple-parallel-susceptor assisted microwave annealing. This relies on the direct energy coupling between non-vanishing dipole moment of inactivated dopant-defect complexes and external electromagnetic field. This interaction between electromagnetic field and $\mathrm{P}_{\mathrm{n}} \mathrm{V}$ is expected to eliminate unstable low-order $\mathrm{P}_{\mathrm{n}} \mathrm{V}(\mathrm{n}=1-3)$, resulting in a specific distribution of the dopant defect dominated by thermally stable $\mathrm{P}_{4} \mathrm{~V}$ clusters. As a result, the dopant deactivation can be effectively suppressed.

This paper is divided into two parts: experimental and theoretical. The experimental part discusses the deactivation phenomena in $\mathrm{Si}: \mathrm{P}$ and thermal stability of $\mathrm{P}$ activation under various thermal annealing treatments. We compared the proposed triple-parallel susceptor microwave annealing (TPS-MWA) treatments to two other well-known approaches: laser annealing (LSA) and flash lamp millisecond annealing (MSA). The effect of dopant deactivation was characterized using sheet resistance measurements $\left(\mathrm{R}_{\mathrm{sh}}\right)$, Hall measurement, and secondary ion mass spectroscopy (SIMS). In the theoretical part, we employed ab-initio calculation to derive formation 
energy, electronic density distribution and dipole moments of $\mathrm{P}_{\mathrm{n}} \mathrm{V}(\mathrm{n}=1-4)$ clusters. The simulation shows non-vanishing dipole moments in $\mathrm{P}_{\mathrm{n}} \mathrm{V}$ structures $(\mathrm{n}=1-3)$, which is key to achieve selective dopant activation through the interaction with external electric field as we proposed in this work.

A 30nm thick, lightly and heavily doped epitaxial Si:P film was grown on a $200 \mathrm{~mm}$ (resistivity $8-12 \Omega \mathrm{cm})<100>$ p-type $\mathrm{Si}$ wafer in a reduced pressure chemical vapor deposition (CVD) chamber (Applied Centura RP Epi $\left.{ }^{\mathrm{TM}}\right)$ with dichlorosilane (DCS) and phosphine $\left(\mathrm{PH}_{3}\right)$ as precursors. The CVD epitaxial Si:P was grown at a temperature of 650 deg $\mathrm{C}$ under a growth pressure of 300 mTorr. $^{3}$ The growth rate of $\mathrm{Si}: \mathrm{P}$ on blanket silicon is about $50 \mathrm{~nm} / \mathrm{hr}$. Rapid thermal annealing (RTA) with temperature 600-950 deg C (Helios® XP RTP, Mattson Technology) and MSA at 1150 deg C (LA-3000-F, Dai Nippon Screen) were used as the benchmarks for the MWA process. For the MWA, the irradiation incorporating with or without impurity doped-SiC susceptors was performed at microwave power of $12 \mathrm{~kW}, 14 \mathrm{~kW}$ and $15 \mathrm{~kW}$ and with dwell times at $110 \mathrm{sec}$ to $300 \mathrm{sec}$ in a prototype microwave annealing chamber (AXOM multimode cavity microwave annealing system, DSG Technologies). It was observed that with our susceptor design, the sample configuration in the susceptor and the microwave power setting were critical to the final dopant activation result. In our samples, we were able to obtain effective annealing for our dopant activation below $700 \mathrm{deg} \mathrm{C}$ only by finding the optimum MWA annealing and choosing an optimal distance of the small spacing between the impurity doped susceptors. While the exact mechanism for microwave-sample-susceptor interaction is not yet completely known, this study reports our findings which demonstrate that an optimal configuration can yield an optimal activation result.

A pyrometer was employed to monitor the susceptor and sample temperature during annealing. To examine the evolution of the electrically active dose, the Si:P samples, including 
pre-and post-annealing cases, were characterized by a four-point probe sheet resistance and Halleffect measurements (Hall 8800, Swin). Dopant atom distributions were analyzed using highresolution secondary ion mass spectrometry (SIMS) (Physical Electronics ADEPT-1010).

For the ab-initio simulations, we used the Vienna ab-initio Simulation Package (VASP) $)^{19,20}$ employing the projector augmented wave (PAW) method ${ }^{21}$ and PBE-PAW pseudopotentials. ${ }^{22}$ Defects were embedded in the cubic supercells with $216 \mathrm{Si}$ atoms. A Si atom was removed from the cell to create a vacancy $(\mathrm{V})$ or replaced by $\mathrm{P}$ to include substitutional $\mathrm{P}$ atoms. The atom positions were relaxed using a conjugate gradient algorithm until the force acting on each atom was less than $0.01 \mathrm{eV}^{-1}$. The volume of the cubic simulation cells was also modified so that the absolute value of the external pressure was lower than $0.05 \mathrm{kBar}$. Periodic boundary conditions were applied in three spatial directions. We used a $450 \mathrm{eV}$ plane-wave kinetic energy cut-off, and a $4 \times 4 \times 4 \Gamma$-centered Monkhorst-Pack k-point mess ${ }^{23}$ for sampling of the Brillouin zone. We considered only neutral charge states in our calculations.

We used the CHARGEMOL program ${ }^{24,25}$ to evaluate the dipole moments of $\mathrm{P}_{\mathrm{n}} \mathrm{V}$ clusters from the electronic density distribution obtained in VASP calculations. This program computed the Density Derived Electrostatics and Chemical (DDEC) net atomic charges and atomic multipoles for periodic and non-periodic systems, which has been proven to properly describe many different systems 26,27

The aim for source-drain junction engineering for sub-3 $\mathrm{nm}$ nanowire FET technology node is to achieve a sheet resistance $\mathrm{R}_{\mathrm{sh}}$ as low as possible and a junction depth $\mathrm{X}_{\mathrm{j}}$ as shallow as 
(a)

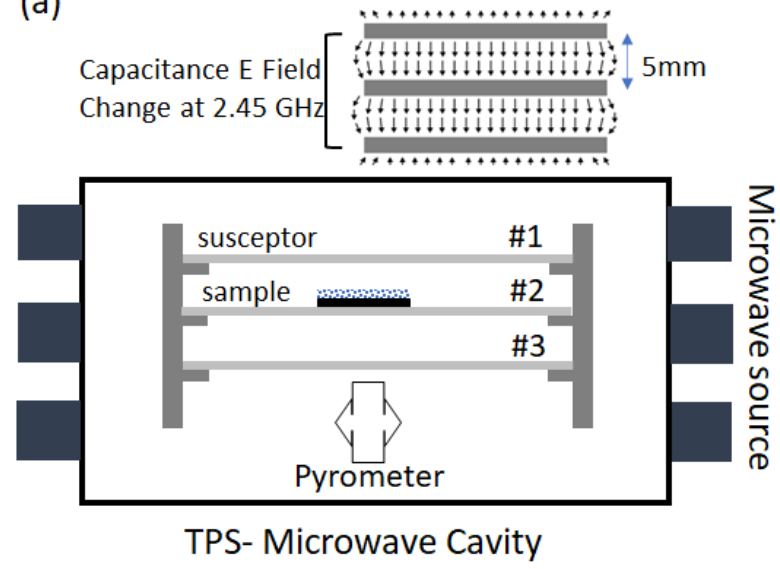

(b)

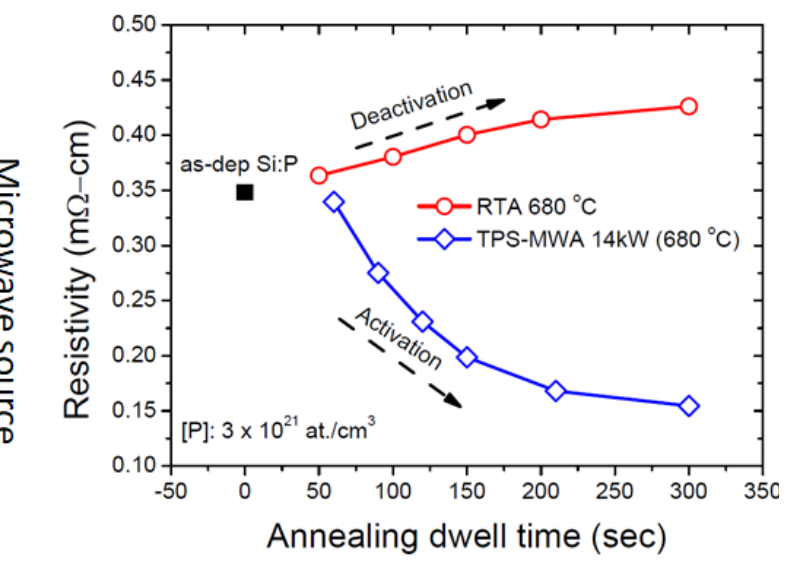

Figure 1. (a) Susceptor setup in a microwave cavity using a triple-susceptor (TPS) configuration where the sample is placed on the middle susceptor with a controlled spacing $5 \mathrm{~mm}$. The inset depicted the E field distribution between susceptor plates. (b) Resistivity of Si:P was measured as a function of the annealing time at $680 \mathrm{deg} C$ at RTA and MWA at $14 \mathrm{~kW}$.

possible. Previously MWA for dopant activation have achieved a comparable $\mathrm{R}_{\mathrm{sh}}-\mathrm{X}_{\mathrm{j}}$ result as compare to isothermal rapid thermal annealing process (RTA). ${ }^{28}$ However, the MWA remain a bulk heating and a long thermal process (> 50sec), the achieved $\mathrm{R}_{\mathrm{sh}}-\mathrm{X}_{\mathrm{j}}$ is therefore constrained by thermal budget and unable to match state-of-the-art millisecond annealing process. ${ }^{29}$ To overcome this thermal budget limitation, our idea is to achieve dopant activation through a direct energy coupling with inactive dopant structures at temperature below $700 \mathrm{deg} \mathrm{C}$. We attempted various susceptor setups in cavity to achieve this goal. Figure. 1 (a) shows an optimum triple-parallelsusceptor setup in the MWA cavity, including the sample holder and susceptor support. The susceptor is comprised of doped Si substrate coated with SiC layer. During MWA process, the temperature of the susceptor rises to target range, thereby the susceptors essentially becomes metallic and behaves as parallel capacitor, each with a specific spacing controlled below $5 \mathrm{~mm}$ from the sample. This configuration leads to a uniform electric field perpendicular to the target sample surface, whereas in the conventional setup without such a susceptor configuration, the 
electromagnetic field of the microwave is expected to be randomly oriented. The triple-parallelsusceptor configuration is critical to allow us to tune the field, and sample temperature to reach a threshold enabling effective direct energy coupling for selective dopant activation.

Fig. 1(b) shows the resistivity as a function of anneal duration. The resistivity of the asdeposited Si:P is $0.35 \mathrm{~m} \Omega$-cm (black symbol), and it monotonically decreased with increased processing time at a constant MWA power of $14 \mathrm{~kW}$, with the substrate temperature kept at 680 $\operatorname{deg} \mathrm{C}$ (as monitored by a pyrometer). Resistivity as low as $0.16 \mathrm{~m} \Omega-\mathrm{cm}$ was obtained after 300 sec in MWA (the blue symbols). The $\mathrm{R}_{\text {sh }}$ trend of Si:P in TPS-MWA process is drastically different from the RTA annealing. As shown the red symbols in Fig. 1(a), using an RTA process that processes equivalent thermal budget as MWA at the same substrate temperature, resistivity is monotonically increasing with time, indicating dopant deactivation instead. This indicates that the dopant activation achieved in the MWA process was not due to a purely thermal effect. In addition, the TPS-MWA treated Si:P sample could be thermally stable.

In heavily doped $\mathrm{Si}$, a high temperature thermal annealing is usually employed to enhance dopant activation level. However, these activated dopants tend to be thermally unstable upon subsequent milder thermal treatments such as RTA. This unstable junction issue is particularly pronounced at high dopant concentration. To better understand the effect of dopant concentration on the deactivation behaviors, Fig 2(a) shows the evolution of the resistivity of Si:P in sequence of thermal annealing for different phosphorus concentration. At lower $[\mathrm{P}]=2 \times 10^{20} \mathrm{at} . / \mathrm{cm}^{3}$, the resistivity remained nearly unchanged before and after thermal sequence, suggesting relatively stable dopant incorporation in Si lattice. In contrast, at higher $[\mathrm{P}]>5 \times 10^{20} \mathrm{at} . / \mathrm{cm}^{3}$ the resistivity of Si:P first decreases after MSA annealing as a result of dopant activation. However, after a subsequent RTA treatment, resistivity increases dramatically due to dopant deactivation. The 
above observation showed that even though Si:P of higher $[\mathrm{P}]$ exhibits significantly lower resistivity after MSA, the donor deactivation effect could lead to a higher final resistivity after RTA. In another words, the activated dopants in highly doped Si are not thermally stable.

(a)

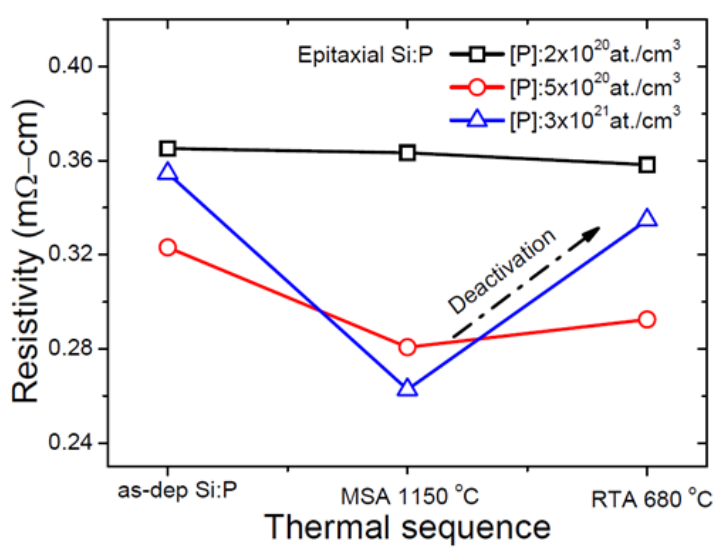

(b)

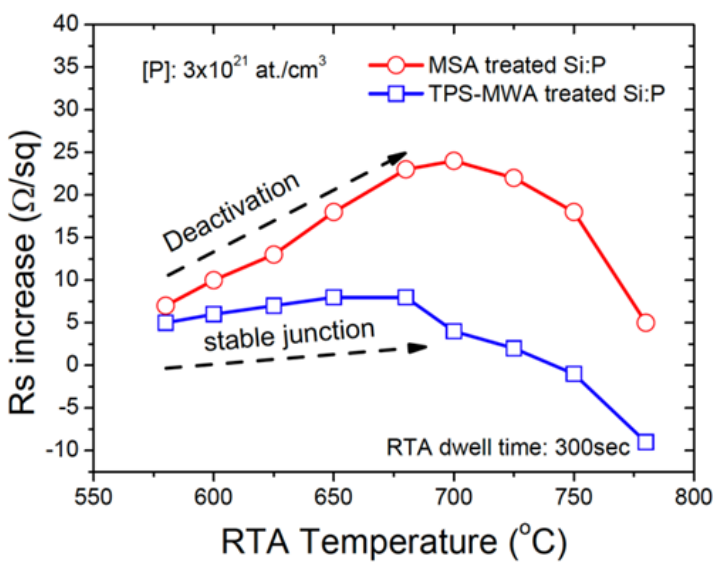

Figure 2. (a) Evolution of resistivity of $\mathrm{Si}: \mathrm{P}$ substrate in sequence of thermal annealing for different phosphorus concentration. (b) Increase in sheet resistance (Rsh) for Si:P samples as a function of RTA annealing temperatures for two different activation processes: TPS-MWA and MSA.

Thermal stability of TPS-MWA annealed Si:P sample at high doping limits was examined by evolution of sheet resistance in a series of thermal annealing at a technologically relevant temperature associated with advanced semiconductor processes. The deactivation thermal annealing was performed for 300s at a temperature range of 600-780 deg C. As shown in Fig. 2(b), the post-microwave annealed samples show relatively small $\mathrm{R}_{\text {sh }}$ changes for $\mathrm{RTA}$ temperature range up to $700 \mathrm{deg} \mathrm{C}$. In contrast, the $\mathrm{R}_{\mathrm{sh}}$ change of the post-MSA annealed sample increased monotonically with increasing RTA temperature up to $700 \operatorname{deg} \mathrm{C}$, where it reached the maximum $R_{\text {sh }}$ value. The above observations indicated that TPS-MWA results in a more thermally stable junction, which is attributed to less dopant deactivation phenomena. To verify this, Hall measurement is used to obtain carrier concentration in these samples. As shown in Fig. 3(a), the 
Si:P samples that are subjected to MSA $1150 \operatorname{deg}$ C and RTA thermal cycles show less carriers, or more pronounced donor deactivation, in accordance to the sheet resistance behavior. In particular, the sample annealed by LSA at a higher temperature at $1225 \operatorname{deg} \mathrm{C}$ showed significant donor deactivation $(\sim 78 \%)$, where the free carrier concentration drops from a super-saturation $8.9 \times 10^{20}$ to $1.9 \times 10^{20} \mathrm{~cm}^{-3}$. In contrast, for TPS-MWA annealed samples, the deactivation amount was only $5 \%$ at $4 \times 10^{20}$, resulting in much higher carrier concentration than those of MSA and LSA counterparts, after the post-thermal treatments.

(a)

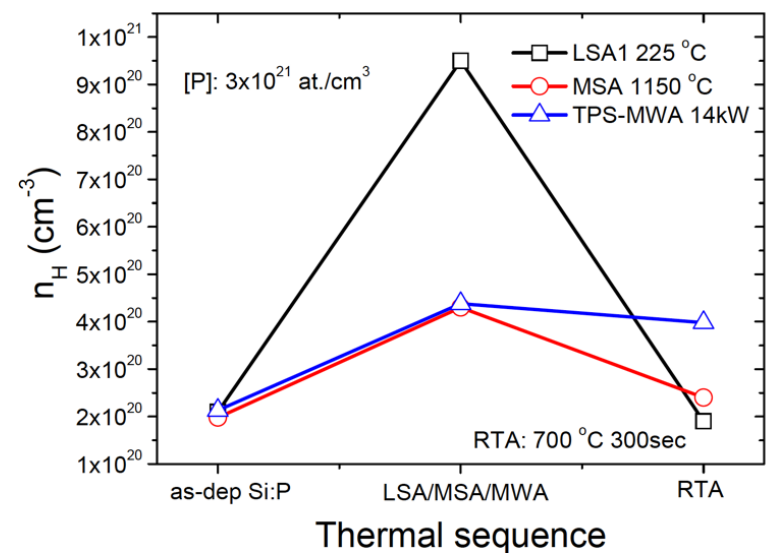

(b)

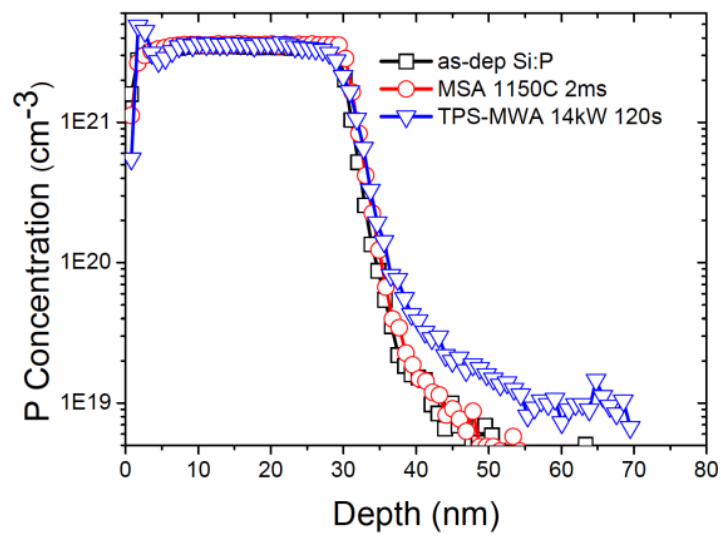

Figure 3. (a) Free carrier concentration change before and after post thermal RTA treatment for various thermally treated Si:P at $3 \times 10^{21}$ at. $/ \mathrm{cm}^{3}$. (b) Phosphorus SIMS profiles in the epitaxial Si:P film after various annealing conditions.

One of the key metrics for Si:P anneal is the dopant diffusion, which needs to be kept at minimal to enable a box-like sharp profile. ToF-SIMS analysis is employed to investigate the doping profile after TPS-MWA, as shown in Fig. 3(b). The results showed a nearly diffusion-less junction at dopant concentration $2 \times 10^{20}$ at. $/ \mathrm{cm}^{3}$ for both MSA and TPS-MWA, indicating that the sheet resistance reduction is mainly attributed to the increase in dopant activation rather than the dopant diffusion. The phosphorus profile of TPS-MWA sample showed a less sharp transition 
compared to MSA annealed sample at concentration below 6E19. This slightly dopant diffusion is believed due to the migration of mobile dopant-vacancy cluster $\left(\mathrm{P}_{1} \mathrm{~V}\right.$ and $\left.\mathrm{P}_{2} \mathrm{~V}\right)$ during isothermal annealing process at temperature $680 \mathrm{deg}$ C under MWA. ${ }^{30,31}$

The above observations suggest that the available pathways for dopant deactivation in Si:P are different for MWA annealed sample compared to LSA and MSA. During MSA and LSA, the UV-visible and near-infrared radiation from the Xeon lamps and $\mathrm{CO}_{2}$ laser are absorbed by the Si:P layer through bandgap and/or free-carrier absorption, which is a non-selective interaction and acts on the bulk of the samples. The thermal annealing of a heavily doped Si:P by MSA or LSA is accompanied by generation of lower order $\mathrm{P}_{\mathrm{n}} \mathrm{V}$ defects. Larsen et al. ${ }^{30}$ showed that annealing of the vacancy-phosphorus pairs could form a new defect associated with a $\mathrm{P}_{2} \mathrm{~V}$ cluster. Vanki et al. ${ }^{31}$ observed that though vacancies can be dissolved from $\mathrm{P}_{\mathrm{n}} \mathrm{V}$ at high temperature, upon cooling the vacancies are quenched to $P_{3} V$ cluster. Among the four kinds of $P_{n} V(n=1-4)$, it appears that $P_{4} V$ possesses the most thermodynamically stable defect as its formation energy is the lowest. ${ }^{17}$ After annealing, these low-order $\mathrm{P}_{\mathrm{n}} \mathrm{V}(\mathrm{n}<4)$ will continue to evolve into a higher order $\mathrm{P}_{\mathrm{n}} \mathrm{V}(\mathrm{n}=3,4)$ at moderate thermal energy and thus result in deactivation of the dopants as shown in Fig. 2(b). Therefore, the post-MSA or LSA samples are thermally unstable due to the presence of these lower order $\mathrm{P}_{\mathrm{n}} \mathrm{V}$ defects.

On the contrary, microwave energy may preferentially interact with vacancy-dopant clusters that possess non-zero dipole moments, whereas the symmetric $\mathrm{P}_{4} \mathrm{~V}$ with a zero net dipole moment is expected to be inert with respect to coupling with microwave energy. Consequently, the $\mathrm{P}_{4} \mathrm{~V}$ dominates the $\mathrm{P}_{\mathrm{n}} \mathrm{V}$ re-distribution in an epitaxial $\mathrm{Si}: \mathrm{P}$ after MWA, and the post-MWA annealed sample would be more thermally stable, as shown in Fig. 2(b). To confirm these dipole-moment 
dependent interactions in MWA, we seek to investigate the detailed electronic characteristics of $\mathrm{P}_{\mathrm{n}} \mathrm{V}$ with $\mathrm{n}=1-4$ by ab-initio simulation.

We performed ab-initio calculation to examine electronic properties, including formation energy and electric dipole moments, of various P-vacancy clusters that are believed to be responsible for most electrically inactive dopants in Si. The atomic configurations considered include the monovacancy $(\mathrm{V})$ configuration, and $\mathrm{P}_{\mathrm{n}} \mathrm{V}$ clusters with $\mathrm{n}=1-4$ where $\mathrm{P}$ atoms were placed at neighboring lattice sites around the vacancy position. We considered $\mathrm{D}_{2 \mathrm{~d}}$ symmetry configurations for the vacancy as it corresponds to the lower energy Si vacancy configuration for the neutral charge state. ${ }^{32}$ The simulation cell size includes $216 \mathrm{Si}$ atoms, and the formation energy of P-vacancy clusters shown in Table. 1 was calculated as indicated in equations 1 and 2 , where $\mathrm{P}_{\mathrm{S}}$ refers to a substitutional $\mathrm{P}$ atom.

$$
\begin{aligned}
& E_{P_{S}}^{f}=E_{T}\left(c S i_{215} P_{S}\right)-E_{T}\left(c S i_{216}\right) \\
& E_{P_{n} V}^{f}=E_{T}\left(P_{n} V\right)-\left\{\frac{215}{216} E_{T}\left(c S i_{216}\right)+n E_{P 1}^{f}\right\}
\end{aligned}
$$

Formation energies are summarized in Table 1, in agreement with previous calculations. ${ }^{17,} 32-$ ${ }^{34}$ Slight differences in the energies arose when the different simulation cell size was adopted (e.g. 64 atoms crystalline $\mathrm{Si}$ simulation cells were used, ${ }^{17,33}$ or a different energy reference for evaluating the formation energy of complexes was employed.$^{33}$ Nevertheless, the high stability of $\mathrm{P}_{4} \mathrm{~V}$ cluster was a common trend in both present and previous theoretical calculations. The negative formation energy of a $\mathrm{P}_{4} \mathrm{~V}$ cluster suggests that its thermal stability should be higher when compared to other lower order $\mathrm{P}_{\mathrm{n}} \mathrm{V}$ clusters. 
Table. 1

Formation energy of studied complexes from Eq. [2].

\begin{tabular}{|c|c|}
\hline Defect & Formation energy \\
\hline $\mathrm{V}(\mathrm{D} 2 \mathrm{~d})$ & $3.63 \mathrm{eV}$ \\
\hline $\mathrm{P}_{1} \mathrm{~V}$ & $2.52 \mathrm{eV}$ \\
\hline $\mathrm{P}_{2} \mathrm{~V}$ & $1.00 \mathrm{eV}$ \\
\hline $\mathrm{P}_{3} \mathrm{~V}$ & $0.10 \mathrm{eV}$ \\
\hline $\mathrm{P}_{4} \mathrm{~V}$ & $-1.43 \mathrm{eV}$ \\
\hline
\end{tabular}

We also evaluated the electronic density distribution in the atomic configurations considered. Fig. 4 shows the electronic density plots of the valence electrons for the $\mathrm{P}_{\mathrm{n}} \mathrm{V}$ clusters. We chose an electronic density reference level of $0.036 \AA^{-3}$ (white color in Fig. 4) to separate the regions with low electronic density (blue regions) from the regions with high electronic density (red regions). Thus, it was easy to identify by visual inspection the electronic density plot atomic positions (dark blue dots which correspond to core regions of atoms as only valence electrons are represented), interstitial spaces (light blue regions) and atomic bonds (intense red regions). Since $\mathrm{P}$ atoms have 5 valence electrons, their location corresponded to the intense red regions indicated by black dashed circles in Fig. 4. There were some atomic rearrangements of dangling bonds around the vacancy in the $\mathrm{P}_{2} \mathrm{~V}$ cluster (Fig. 4.b), which made the Si atoms move out of the plotted plane and the corresponding dark blue dots do not appear in the representation. It can be seen from Fig. 4 that there was a lower electronic density at the vacancy region as more $\mathrm{P}$ atoms are in the cluster (blue region at the surroundings of the vacancy position). As expected, the electron density distribution showed asymmetric feature except for the $\mathrm{P}_{4} \mathrm{~V}$ cluster (Fig. 4.d). This indicated the existence of a non-vanishing dipole moment in the $\mathrm{P}_{\mathrm{n}} \mathrm{V}(\mathrm{n}=1-3)$ clusters. In particular, due to the 
symmetry of the charge distribution in the $\mathrm{P}_{4} \mathrm{~V}$ cluster, its net dipole moment was negligible. This was not the case for other $\mathrm{P}_{\mathrm{n}} \mathrm{V}$ clusters where the dipole moment points in the direction away from the $\mathrm{P}$ atoms and towards their first Si neighbors. The dipole moments for each $\mathrm{P}_{\mathrm{n}} \mathrm{V}$ are depicted and summarized in Fig. 5.

This dipole moment can couple to the external electric fields which can induce charge oscillations that eventually result in localized atomic movements in the vicinity of the $\mathrm{P}_{\mathrm{n}} \mathrm{V}$ clusters with net dipole moments. Depending on the dipole moment orientation of the $\mathrm{P}_{\mathrm{n}} \mathrm{V}$ cluster, the selective energy coupling with microwave was found to result in selective dopant activation at a microscopic level within the localized lattice of the film.

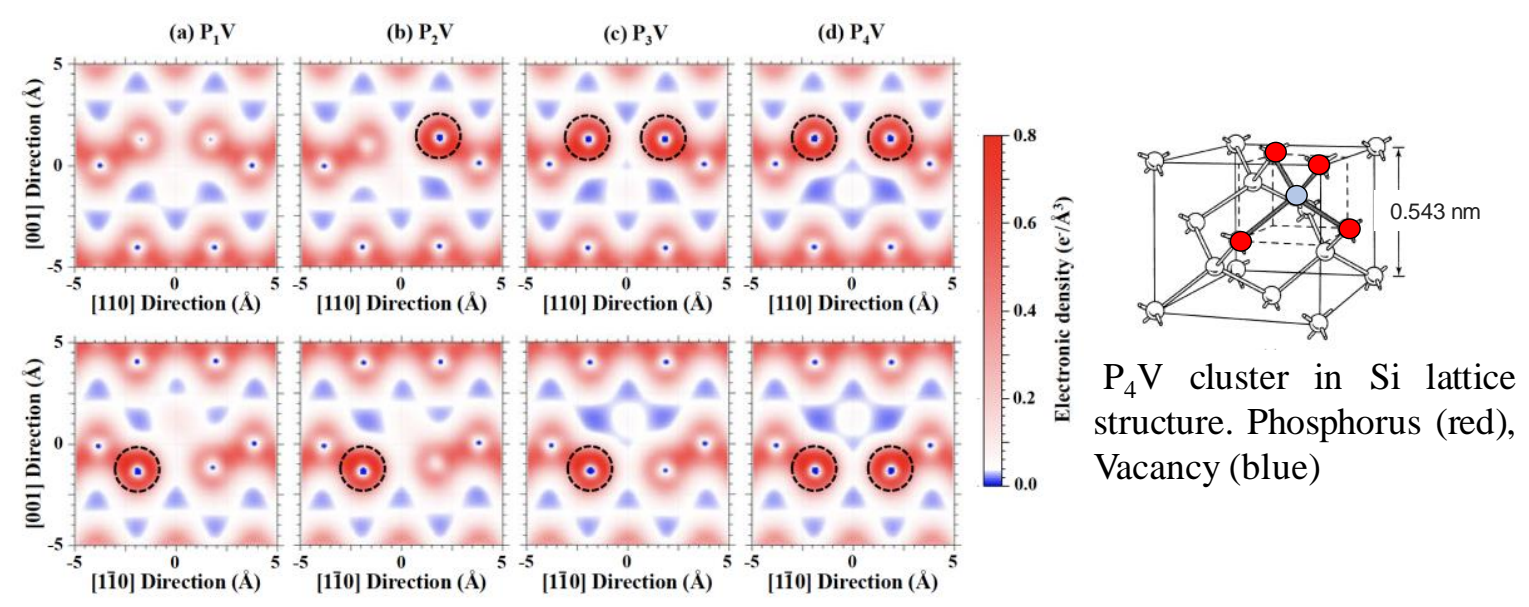

Figure 4. Electronic density plots of valence electrons for $\mathrm{P}_{\mathrm{n}} \mathrm{V}$ complexes. The origin of plots is located at the center of the plot and corresponds to the position of the vacancy before relaxing the atomic configuration. Black dashed circles indicate the location of P atoms. Inset plot shows a $\mathrm{P}_{4} \mathrm{~V}$ cluster in Si lattice structure.

Through the experimental results and theoretical calculation, we believe that the superior thermal stability feature with TPS-MWA annealed samples was due to a different $\mathrm{P}_{\mathrm{n}} \mathrm{V}$ distribution dominated by the $\mathrm{P}_{4} \mathrm{~V}$ cluster. In TPS-microwave annealing process, the average sample temperature was maintained through background dielectric heating within bulk substrate 

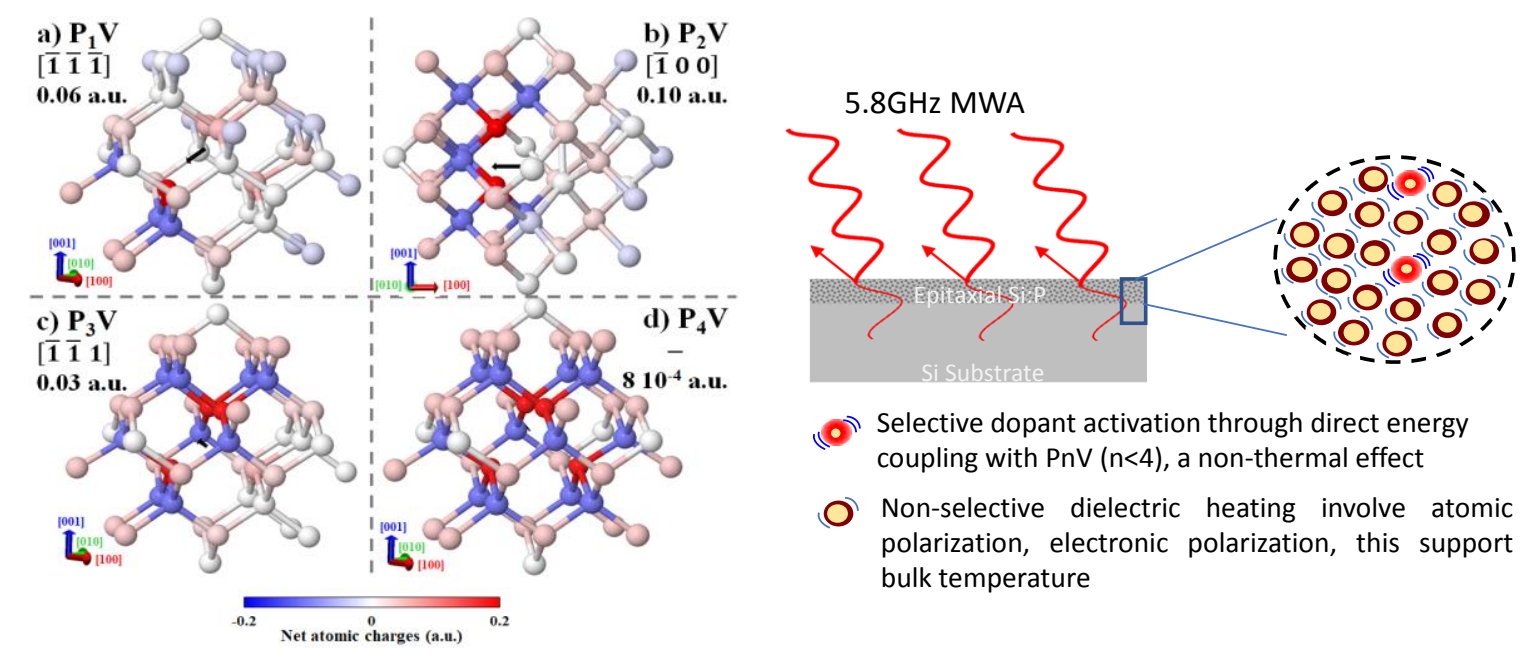

Selective dopant activation through direct energy coupling with $\mathrm{PnV}(\mathrm{n}<4)$, a non-thermal effect

(O) Non-selective dielectric heating involve atomic polarization, electronic polarization, this support bulk temperature

Figure 5. Net atomic charges (color scale) and dipole moments (black arrows) of PnV clusters obtained from the electronic density distribution using CHARGEMOL program. Dipole moment direction and magnitude are indicated. $\mathrm{P}$ atoms have larger positive charges (intense red atoms), while first Si neighbors of $\mathrm{P}$ atoms have a larger negative charge (intense blue atoms). For better visualization, only atoms up to $6 \AA$ from the vacancy position are shown and net atomic dipole moments are centered at the vacancy position. Inset depicted selective dopant activation through direct energy coupling with the non-vanishing dipole moment in $\mathrm{P}_{\mathrm{n}} \mathrm{V}(\mathrm{n}<4)$ as a contrast to the non-selective dielectric heating that support bulk temperature of sample

(inset of Fig. 5). It was obvious that the observed dopant activation cannot be accounted for in such low background temperature below $700 \mathrm{deg}$ C. On the contrary, the fact that a high doping activation compare to MSA annealing temperature at $1150 \mathrm{deg} \mathrm{C}$ indicates that a highly efficient coupling to the microwave was present for the dopant vacancy cluster. As depicted in the inset of Fig. 5, this activation appears to be a selective process in nature due to the non-zero dipole moment of the $P_{n} V$ structures $(n=1-3)$. This result also suggested that the evolution of lower order $P_{n} V$ clusters toward energetically favored $\mathrm{P}_{4} \mathrm{~V}$ structures at moderate temperatures (600-700 $\operatorname{deg} \mathrm{C}$ ) was responsible for the so-called dopant deactivation phenomenon widely observed in the highly doped Si:P sample. We believe that the phenomenon of the donor deactivation suppression as observed in our study, can also be seen for other donors such as Arsenic (As), Antimony (Sb) and 
other substrate material such as $\mathrm{Ge}$ and $\mathrm{SiGe}$ since the dominant donor deactivation mechanism for such donors (e.g. P, As, Sb) appear to be associated with the formation of dopant-vacancy clusters. ${ }^{17,35,36}$

In summary, we have demonstrated that with the assistance of an optimum triple-parallelsusceptor set-up and optimal sample configuration, a direct energy coupling effect in microwave annealing process can be achieved. This unique feature is able to achieve selective dopant activation in heavily doped Si:P at a low temperature below $700 \mathrm{deg}$ C. For the first time, the obtained shallow junction not only can provide high carrier concentration compared to state-ofthe-art MSA and LSA process typically achieved at temperatures above $1100 \mathrm{deg} \mathrm{C}$, but it can also offer superior thermal stability for activated dopants by eliminating lower-order $\mathrm{P}_{\mathrm{n}} \mathrm{V}$ clusters. This indicates that controlling the lower order donor vacancy cluster distribution within a heavily doped Si layer is a key important factor in overcoming the well-known donor deactivation limitation for semiconductor application.

\section{ACKNOWLEDGMENTS}

The authors would like to thank H. -J. Gossmann from Applied Materials, Paul Timans from Mattson Technology, and Yu-Ming Lin from Taiwan Semiconductor Manufacturing Conmpany for fruitful discussion on the thermal and non-thermal activation mechanism. We would also like to acknowledge funding from Taiwan's Ministry of Science and Technology under Contract Number MOST-109-2628-M-008-004-MY3.

\section{DATA AVAILABILITY STATEMENT}


The data that support the findings of this study are available from the corresponding author upon reasonable request.

\section{REFERENCES}

[1] C. N. Ni, X. Li, S. Sharma, K.V. Rao, M. Jin, C. Lazik, V. Banthia, B. Colombeau, N. Variam, A. Mayur, H. Chung, R. Hung, and A. Brand, Ultra-low contact resistivity with highly doped Si:P contact for nMOSFET, IEEE Symp. IEEE Symp. VLSI Technol. T118 (2015).

[2] C. N. Ni, K.V. Rao, F. Khaja, S. Sharma, S. Tang, J. J. Chen, K. E. Hollar, N. Breil, X. Li, M. Jin, C. Lazik, J. Lee, H. Maynard, N. Variam, A.J. Mayur, S. Kim, H. Chung, M. Chudzik, R. Hung, N. Yoshida, and N. Kim, Ultra-low NMOS contact resistivity using a novel plasmabased DSS implant and laser anneal for post $7 \mathrm{~nm}$ nodes, IEEE Symp. VLSI Technol. 1 (2016).

[3] X. Li, A. Dube, Z. Ye, S. Sharma, Y. Kim, and S. Chu, Selective epitaxial Si:P film for nMOSFET application: high phosphorous concentration and high tensile strain, ECS Trans. 64, 959-965 (2014).

[4] S. K. Dhayalan, J. Kujala, J. Slotte, G. Pourtois, E. Simoen, E. Rosseel, A. Hikavyy, Y. Shimura, S. Iacovo, A. Stesmans, R. Loo, and W. Vandervorst, On the manifestation of phosphorusvacancy complexes in epitaxial Si:P films, Appl. Phys. Lett. 108, 082106 (2016).

[5] N. Singh, K. D. Buddharaju, S. K. Manhas, A. Agarwal, S. C. Rustagi, G. Q. Lo, N. Balasubramanian, and D.-L. Kwong, Si, SiGe Nanowire Devices by Top-Down Technology and Their Applications, IEEE Trans. Electron. Dev. 55, 3107 (2008).

[6] S. D. Suk, S.-Y. Lee, S.-M. Kim, E.-J. Yoon, M.-S. Kim, M. Li, C. W. Oh, K. H. Yeo, S. H. Kim, D.-S. Shin, K.-H. Lee, H. S. Park, J. N. Han, C. J. Park, J.-B. Park, D.-W. Kim, D. Park, 
and B.-I. Ryu, High performance $5 \mathrm{~nm}$ radius twin silicon nanowire MOSFET(TSNWFET): Fabrication on bulk Si wafer, characteristics and reliability, IEDM Tech. Dig. 717 (2005)

[7] H. Mertens, R. Ritzenthaler, A. Chasin, T. Schram, E. Kunnen, A. Hikavyy, L.-Å. Ragnarsson, H. Dekkers, T. Hopf, K. Wostyn, K. Devriendt, S. A. Chew, M. S. Kim, Y. Kikuchi, E. Rosseel, G. Mannaert, S. Kubicek, S. Demuynck, A. Dangol. N. Bosman, J. Geypen, P. Carolan, H. Bender, K. Barla, N. Horiguchi, and D. Mocuta, Vertically Stacked Gate-All-Around Si Nanowire CMOS Transistors with Dual Work Function Metal Gates, IEDM Tech. Dig. 524 (2016).

[8] M. T. Björk, H. Schmid, J. Knoch, H. Riel, and W. Riess, Donor deactivation in silicon nanostructures Nature Nanotechnology, Nat. Nanotechnol. 4, 103 (2009).

[9] K. C. Pandey, A. Erbil, G. S. Cargill, R. F. Boehme, and D. Vanderbilt, Annealing of Heavily Arsenic-Doped Silicon: Electrical Deactivation and a New Defect Complex, Phys. Rev. Lett. $61,1282(1988)$.

[10] P. M. Fahey, P. Griffin, and J. D. Plummer, Point defects and dopant diffusion in silicon, Rev. Mod. Phys. 61, 289 (1989).

[11] P. A. Packan, Pushing the Limits, Science. 285, 2079 (1999).

[12] A. N. Larsen, K. K. Larsen, and P. E. Andersen, Heavy doping effects in the diffusion of group IV and V impurities in silicon, J. Appl. Phys. 73, 691 (1993).

[13] Y. Takamura, S. H. Jain, P. B. Griffin, and J. D. Plummer, Thermal stability of dopants in laser annealed silicon, J. Appl. Phys. 92, 230 (2002).

[14] Y. Takamura, P. B. Griffin, and J. D. Plummer, Physical processes associated with the 
deactivation of dopants in laser annealed silicon, J. Appl. Phys. 92, 235 (2002).

[15] V. Ranki, K. Saarinen, J. Fage-Pedersen, J. L. Hansen, and A. N. Larsen, Electrical deactivation by vacancy-impurity complexes in highly As-doped Si, Phys. Rev. B. 67, 041201(2003).

[16] D. C. Mueller, and W. Fichtner, Highly n-doped silicon: Deactivating defects of donors, Phys. Rev. B. 70, 245207 (2004).

[17] R. Chen, B. Trzynadlowski, and S. T. Dunham, Phosphorus vacancy cluster model for phosphorus diffusion gettering of metals in Si, J. Appl. Phys. 115, 054906 (2014).

[18] S. Prucnal, L. Rebohle, and W. Skorupa, Doping by flash lamp annealing, Materials Science in Semiconductor Processing. 62, 115 (2017).

[19] G. Kresse, and J. Furthmüller, Efficient iterative schemes for ab initio total-energy calculations using a plane-wave basis set, Phys. Rev. B. 54, 11169 (1996).

[20] G. Kresse, and J. Furthmüller, Efficiency of ab-initio total energy calculations for metals and semiconductors using a plane-wave basis set, Comput. Mater. Sci. 6, 15 (1996).

[21] G. Kresse, and D. Joubert, From ultrasoft pseudopotentials to the projector augmented-wave method, Phys. Rev. B. 59, 1758 (1999).

[22] J. P. Perdew, K. Burke, and M. Ernzerhof, Generalized Gradient Approximation Made Simple, Phys. Rev. Lett. 77, 3865 (1996).

[23] H. J. Monkhorst, and J. D. Pack, Special points for Brillouin-zone integrations, Phys. Rev. B $13,5188(1976)$.

[24] N. G. Limas, and T. A. Manz, Introducing DDEC6 atomic population analysis: part 2. 
Computed results for a wide range of periodic and nonperiodic materials, RSC Adv. 6, 45727 (2016).

[25] T. A. Manz, and N. Gabaldon, Introducing DDEC6 atomic population analysis: part 1. Charge partitioning theory and methodology, RSC Adv. 6, 47771 (2016).

[26] T. A. Manz, Introducing DDEC6 atomic population analysis: part 3. Comprehensive method to compute bond orders, RSC Advances. 7, 45552 (2017).

[27] N. G. Limas, and T. A. Manz, Introducing DDEC6 atomic population analysis: part 4. Efficient parallel computation of net atomic charges, atomic spin moments, bond orders, and more, RSC Advances. 8, 2678 (2018).

[28] P. Xu, C. Fu, C. Hu, D. W. Zhang, D. Wu, J. Luo, C. Zhao, Z.-B. Zhang, S.-L. Zhang, Appl. Phys. Lett. 102, 122114 (2013)

[29] L. Rebohle, S. Prucnal, and D. Reichel, Flash Lamp Annealing: From Basics to Applications, Springer Nature, Swizerland, ISBN: 978-3-030-23298-6 P136 (2019).

[30] A. N. Larsen, K. K. Larsen, P. E. Andersen. B. G. Svensson, J. Appl. Phys. 73, 691 (1993).

[31] V. Ranki, K. Saarinen, Phys. Rev. Lett. 93, 255502 (2004).

[32] J. Dabrowski and G. Dissinger, Supercell-size convergence of formation energies and gap levels of vacancy complexes in c-Si in DFT calculations, Phys. Rev. B 92, 144104 (2015).

[33] SM. Lee, H.-Y. Riu, E. Ko , D.-H. Ko, Effects of P doping and post-growth laser annealing on the structural, electrical an chemical properties of P-doped Si films, ACS Appl. Electron. Mater. 1, 288 (2019). 
[34] B. Sahli, K. Vollenweider, W. Fichtner, Ab initio calculations for point defect clusters with P As, and Sb in Si, Phys. Rev. B 80, 075208 (2009).

[35] A. Chroneosa, Dopant-vacancy cluster formation in germanium, Dopant-vacancy cluster formation in germanium, J. Appl. Phys. 107, 076102 (2010).

[36] A. Chroneos, and H. Bracht, Diffusion of n-type dopants in germanium, Diffusion of n-type dopants in germanium, Appl. Phys. Rev. 1, 011301 (2014). 